\title{
Dp-brane Tension from Tachyons and B-field in Vacuum String Field Theory
}

\author{
P. Matlock ${ }^{a}$, R.C. Rashkov ${ }^{b}$, K.S. Viswanathan ${ }^{a}$ and Y. Yang ${ }^{a}$ \\ ${ }^{a}$ Department of Physics, Simon Fraser University, \\ Burnaby BC, Canada \\ pwm@sfu.ca, kviswana@sfu.ca, yyangc@sfu.ca \\ ${ }^{b}$ Department of Physics, Sofia University \\ 1164 Sofia, Bulgaria \\ rash@phys.uni-sofia.bg \\ March $7^{\text {th }}, 2002$
}

\begin{abstract}
We consider tachyonic string-field fluctuations about a $\mathrm{D} p$-brane background in the geometrical (CFT) formulation of Vacuum String Field Theory. We then extend this analysis to the case of a background $B$-field. We find that the standard results for D-brane tension are reproduced in both cases.
\end{abstract}




\section{Introduction}

Vacuum String Field Theory [14 is believed to represent Witten's cubic open string field theory [1] (see also [2, 3, 4, 5, 6]) near the tachyon vacuum. Sen conjectured that unstable D-brane configurations may collapse into other stable configurations via condensation of the tachyon [7, 8]. In particular, the D25-brane background of open bosonic string theory is expected to condense into a non-perturbative tachyon vacuum [9, 10]. In VSFT the usual BRST operator $Q$, whose cohomology classes represent physical states in the full theory, is replaced by a new operator $\mathcal{Q}$ which operates only on ghost degrees of freedom and has trivial cohomology. Therefore there are no perturbative physical states in VSFT, and moreover due to this simplification it is possible to find classical solutions to the string-field equation of motion [4, 5, 11, 14. In Witten's original notation [1], the action is given by

$$
S[\Psi]=-\frac{1}{2} \int\left(\Psi \star Q \Psi+g \frac{2}{3} \Psi \star \Psi \star \Psi\right),
$$

so that the equation of motion for the string-field $\Psi$ is $Q \Psi=\Psi \star \Psi$.

The problem of tachyon fluctuations and the resulting D25-brane tension in the context of VSFT was first considered by Hata and Kawano [20], and by Rastelli, Sen and Zwiebach [15]. In the former paper the D25-brane tension did not seem to be compatible with the standard result, and in the latter doubt was expressed as to the validity of the linearised equations of motion for the tachyon perturbation, when the BPZ-product with the perturbation state (which is slightly outside the Fock-space) is taken. The analysis by two of the present authors in [18] clarified the limiting procedure involved when taking star- and BPZ-products, and showed that the linearised equation of motion is indeed valid in the 'strong' sense, obtaining a result for the D25-brane tension compatible with the assertion that the sliver solution represents a single D25-brane.

Here we consider tachyon fluctuations about a $\mathrm{D} p$-brane solution for arbitrary $p$ and in this way evaluate the tension of the D-brane. We make use of boundary condition-changing twist operators, as suggested in [13], in the 'geometrical' conformal field theory approach. We find the correct ratios of tensions between branes of differing dimension. Our results may be compared to those found in the recent paper [17] which were obtained using the algebraic (oscillator) [19, 2, 3] approach. We also investigate the effect of a $B$-field and again obtain the expected ratios of tensions.

This paper is organised as follows. In section 2 we discuss the construction of perturba- 
tive states around a D-brane solution, in particular focussing our attention on the tachyon field. Next, in section 3 we construct this perturbative tachyon state around a $\mathrm{D} p$-brane configuration and evaluate the resulting tension. Section 1 contains an analysis of the effect of a $B$-field background, and we again obtain the standard expression for the tension of a non-commutative D-brane. We conclude with some comments in section 5 .

\section{D $p$-brane}

In Fock-space notation, as used in the geometrical approach of Rastelli, Sen and Zwiebach [14, the action is given by

$$
S[\Psi]=-\kappa\left[\frac{1}{2}\langle\Psi \mid \mathcal{Q} \Psi\rangle+\frac{1}{3}\langle\Psi \mid \Psi * \Psi\rangle\right],
$$

and the equation of motion is written

$$
\mathcal{Q}|\Psi\rangle=|\Psi * \Psi\rangle
$$

In VSFT the operator $\mathcal{Q}$ affects only the ghost sector of the theory; the equation of motion thus factorises and the matter part becomes

$$
\left|\Psi_{m}\right\rangle=\left|\Psi_{m} * \Psi_{m}\right\rangle
$$

It is conjectured that the ghost part of the solution is universal [7].

One solution $\Xi$ to the matter equation of motion, called the sliver state, is believed to correspond to a D25-brane. This state is defined by

$$
\langle\Xi \mid \phi\rangle=\lim _{n \rightarrow \infty} \mathcal{N}\langle f \circ \phi(0)\rangle_{C_{n}}
$$

where on the RHS the brackets denote a correlation function in the matter BCFT on the space $C_{n} . C_{n}$ is taken to be the semi-infinite cylinder obtained by identifying $\Re e z \equiv \Re e z+n \pi / 2$ in the complex $z$ upper half plane. $\phi$ is an operator representing an arbitrary state in the Fock space and $|\phi\rangle$ is the corresponding state obtained by inserting $\phi$ at $z=0$ in the 'local patch' $-\pi / 4<\Re e z<\pi / 4$. We refer the reader to [14] for details of this construction.

In order to extend this to the case of a D $p$-brane, we need to introduce boundary condition-changing twist operators [13, 19]. These are $\sigma^{+}$and $\sigma^{-}$and are inserted at $z= \pm \pi / 4 \pm \epsilon$. This effectively imposes Neumann boundary conditions on $-\pi / 4<z<\pi / 4$ 
and Dirichlet conditions on the rest of the boundary, $\pi / 4<z<(2 n-1) \pi / 4$. The correlator in eqn. (4) is taken over the 26 independent conformal fields $X^{\mu}$. In this case, we define

$$
\langle\mathrm{D} p \mid \phi\rangle=\lim _{n \rightarrow \infty} \mathcal{N}\langle f \circ \phi(0)\rangle_{C_{n}}^{\sigma \perp},
$$

where the superscript indicates the presence of the $\sigma$ operators in the $25-p$ Dirichlet directions, while the $p+1$ Neumann directions are unchanged. The state $|\mathrm{D} p\rangle$ so defined represents a $\mathrm{D} p$-brane [13] and it satisfies the equation of motion (2), provided that it is renormalised as follows. When we take the star-product of the sliver with itself, we will obtain a short-distance singularity from the proximity of $\sigma^{+}$from one sliver, and $\sigma^{-}$from the other. As noted in [13], the leading term of the operator expansion will have no operator content, and so this singularity will only contribute $(1 / 2 \epsilon)^{h}$ to the product, where $h$ depends on the conformal dimensions of the $\sigma$-operators. This divergent factor may simply be absorbed into the definition of the sliver state.

\section{$3 \quad$ VSFT Perturbations and $\mathbf{D} p$-brane Tension}

We wish to start with a background string-field solution $\Phi_{0}=\Phi_{\text {ghost }} \otimes \Phi$ and consider perturbations parametrised by fields. We follow here the procedure used in [18], generalised to a the case of a D p-brane. As in [15], we use the perturbative expansion

$$
|\Psi\rangle=\left|\Phi_{g}\right\rangle \otimes\{|\Phi\rangle+|T\rangle+\cdots\}
$$

where $|T\rangle$ is a tachyon excitation and terms corresponding to vector and higher excitations follow. The tachyon perturbation is

$$
|T\rangle=\int \mathrm{d} k n^{-k_{\|}^{2}} T(k)|\chi(k)\rangle
$$

with $T(k)$ the momentum-space tachyon field. We use $k_{\|}$to refer to directions longitudinal to the D-brane, and $k_{\perp}$ for transverse directions.

We may insert this expansion for $|\Psi\rangle$ into the action (1) to obtain

$$
S[T]=S\left[\Phi_{g} \otimes \Phi\right]-\left\langle\Phi_{g} \mid \mathcal{Q} \Phi_{g}\right\rangle\left[\frac{1}{2}\langle T \mid T\rangle-\langle\Phi \mid T * T\rangle+\frac{1}{3}\langle T \mid T * T\rangle\right]
$$

whence the linearised equation of motion for $|T\rangle$ can be obtained;

$$
|\chi(k)\rangle=|\chi(k) * \Phi\rangle+|\Phi * \chi(k)\rangle .
$$


In this section we take as the background solution the $\mathrm{D} p$-brane state $|\Phi\rangle=|\mathrm{D} p\rangle$. It was shown in [18] that the linearised equation of motion for a perturbation $\chi(k)$ about the sliver state holds even when the BPZ-product with another solution $\chi\left(k^{\prime}\right)$ is taken, that is

$$
\left\langle\chi(k) \mid \chi\left(k^{\prime}\right)\right\rangle=\left\langle\chi(k) * \Xi+\chi(k) * \Xi \mid \chi\left(k^{\prime}\right)\right\rangle .
$$

Equation (9) has been referred to as the 'weak' equation of motion, with eqn. (10) being a stronger version, since $\Xi$ and thus $\chi$ are not quite Fock space states.

The linearised equation of motion (9) for a tachyon perturbation $\chi(k)$ about the sliver state $\Xi$ was solved in [20] and this solution was expressed in the CFT language in [15]. In the present case of a $\mathrm{D} p$-brane, the solution may be written

$$
\langle\chi(k) \mid \phi\rangle=\lim _{n \rightarrow \infty} \mathcal{N} n^{2 k^{2}}\left\langle f \circ \phi(0) e^{i k \cdot X}(n \pi / 4)\right\rangle_{C_{n}}^{\sigma \perp},
$$

so that the momentum degrees of freedom are carried by a tachyon vertex operator inserted diametrically opposite the $\phi$-insertion puncture. The factor $n^{2 k_{\|}^{2}}$ is inserted to compensate for a factor $n^{-2 k_{\|}^{2}}$ which will come from the correlator.

Let us now show that the state in eqn. (11) satisfies the equation of motion (10). Computing first the LHS, we find

$$
\left\langle\chi(k) \mid \chi\left(k^{\prime}\right)\right\rangle=\mathcal{N}^{2} \lim _{n, m \rightarrow \infty} n^{2 k^{2}} m^{2 k^{\prime} \|}\left\langle e^{i k \cdot X}(0) e^{i k^{\prime} \cdot X}\left((n+m-2) \frac{\pi}{4}\right)\right\rangle_{C_{m}+n-2}^{\sigma \perp} .
$$

This is calculated in the appendix and is given by eqn. (A-6),

$$
\left\langle\chi(k) \mid \chi\left(k^{\prime}\right)\right\rangle=\mathcal{K} \lim _{n \rightarrow \infty} n^{2 k_{\|}^{2}} 2^{2 k_{\|}^{2}}(2 \pi)^{26} \delta\left(k_{\|}+k_{\|}^{\prime}\right) e^{i x_{0} \cdot\left(k_{\perp}+k_{\perp}^{\prime}\right)} .
$$

Shifting our attention to the RHS of eqn. (10), we may express either of the two terms as

$$
\left\langle\Xi * \chi(k) \mid \chi\left(k^{\prime}\right)\right\rangle=\mathcal{N}^{2} \lim _{n_{1}, n_{2}, n_{3} \rightarrow \infty} n_{2}^{2 k_{\|}^{2}} n_{3}^{2 k^{\prime} \|}\left\langle e^{i k \cdot X}(0) e^{i k^{\prime} \cdot X}\left(\left(n_{2}+n_{3}-2\right) \frac{\pi}{4}\right)\right\rangle_{C_{n_{1}+n_{2}+n_{3}-3}}^{\sigma \perp} .
$$

Evaluating this correlator in the same way we find

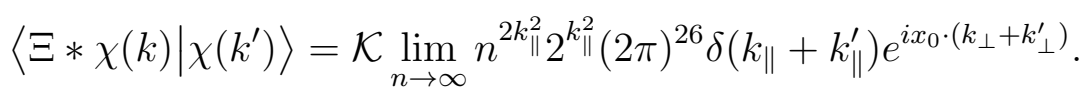

Substituting eqn. (13) and eqn. (15) into the equation of motion (10), it reduces to

$$
k_{\|}^{2}=1
$$


We see that the tachyon living on the $\mathrm{D} p$-brane satisfies the strong equation of motion, provided that it is on-shell. This is to be compared with the case of a D25-brane [18]. Off-shell we have

$$
2^{1-k_{\|}^{2}}\left\langle\chi(k) \mid \chi\left(k^{\prime}\right)\right\rangle=\left\langle\chi(k) * \Xi+\chi(k) * \Xi \mid \chi\left(k^{\prime}\right)\right\rangle .
$$

To calculate the $\mathrm{D} p$-brane tension, we first examine the quadratic term $S^{(2)}$ of the action (8) to fix the normalisation of the tachyon field $T(k)$. Using eqn. (17) we may write

$$
S^{(2)}=-\frac{1}{2}\left\langle\Psi_{g} \mid \mathcal{Q} \Psi_{g}\right\rangle \int \mathrm{d} k \mathrm{~d} k^{\prime} T(k) T\left(k^{\prime}\right)\left(1-2^{1-k_{\|}^{2}}\right)\left\langle\chi(k) \mid \chi\left(k^{\prime}\right)\right\rangle .
$$

After substituting the product from eqn. (13), we have

$$
\begin{aligned}
S^{(2)}= & -\frac{1}{2}\left\langle\Psi_{g} \mid \mathcal{Q} \Psi_{g}\right\rangle \mathcal{K} \int \mathrm{d}^{p+1} k_{\|} \mathrm{d}^{p+1} k_{\|}^{\prime}\left(1-2^{1-k_{\|}^{2}}\right) 2^{k_{\|}^{2}}(2 \pi)^{26} \delta\left(k_{\|}+k_{\|}^{\prime}\right) \\
& \left\{\int \mathrm{d}^{25-p} k_{\perp} \mathrm{d}^{25-p} k_{\perp}^{\prime} e^{i x_{0} \cdot\left(k_{\perp}+k_{\perp}^{\prime}\right)} T(k) T\left(k^{\prime}\right)\right\}
\end{aligned}
$$

Taking $T(k)$ near on-shell, $k_{\|}^{2} \approx 1$, we may write the $k_{\|}$-dependent factor as $\left(k_{\|}^{2}-1\right) 4 \log 2$. We see that a re-definition of the tachyon field

$$
T_{\mathrm{D} p}\left(k_{\|}\right) \equiv \sqrt{\mathcal{K}(2 \pi)^{25-p}\left\langle\Psi_{g} \mid \mathcal{Q} \Psi_{g}\right\rangle 4 \log 2} \int \mathrm{d}^{25-p} k_{\perp} e^{i x_{0} \cdot k_{\perp}} T(k)
$$

would cast $S^{(2)}$ into the canonical form

$$
S^{(2)}=-\frac{1}{2}(2 \pi)^{p+1} \int \mathrm{d}^{p+1} k_{\|}\left(k_{\|}^{2}-1\right) T_{\mathrm{D} p}\left(k_{\|}\right) T_{\mathrm{D} p}\left(-k_{\|}\right) .
$$

The cubic term in the action involves

$$
\begin{aligned}
& \left\langle\chi\left(k_{1}\right) \mid \chi\left(k_{2}\right) * \chi\left(k_{3}\right)\right\rangle \\
& =\left\langle e^{i k_{1} \cdot X}(0) e^{i k_{2} \cdot X}\left(\left(n_{1}+n_{2}-2\right) \frac{\pi}{4}\right) e^{i k_{3} \cdot X}\left(-\left(n_{1}+n_{3}-2\right) \frac{\pi}{4}\right)\right\rangle_{C_{n_{1}+n_{2}+n_{3}-3}}^{\sigma \perp}
\end{aligned}
$$

Once again mapping to the unit disc, we may use Wick's theorem and the tachyon correlator from eqn. (A-4) to find

$$
\left\langle\chi\left(k_{1}\right) \mid \chi\left(k_{2}\right) * \chi\left(k_{3}\right)\right\rangle=\lim _{n \rightarrow \infty} 2 n^{\sum k_{\|}^{2}}(2 \pi)^{26} e^{i x_{0} \cdot\left(\sum k_{\perp}\right)} \delta\left(\sum k_{\|}\right)
$$


Substituting this result into the action, the cubic term $S^{(3)}$ is

$$
\begin{aligned}
S^{(3)}= & -\frac{2}{3}\left\langle\Psi_{g} \mid \mathcal{Q} \Psi_{g}\right\rangle \mathcal{K} \int \mathrm{d} k_{1,2,3 \|}(2 \pi)^{26} \delta\left(\sum k_{\|}\right) \\
& \left\{\int \mathrm{d} k_{1,2,3 \perp} T\left(k_{1}\right) T\left(k_{2}\right) T\left(k_{3}\right) e^{i x_{0} \cdot\left(\sum k_{\perp}\right)}\right\},
\end{aligned}
$$

which we can write in terms of the D-brane tachyon field (20) as

$$
\begin{aligned}
S^{(3)=} & -\frac{1}{3} \frac{2(2 \pi)^{p+1}}{(4 \log 2)^{3 / 2} \sqrt{\mathcal{K}(2 \pi)^{p-25}\left\langle\Psi_{g} \mid \mathcal{Q} \Psi_{g}\right\rangle}} \\
& \int \mathrm{d} k_{1,2,3 \|} T_{\mathrm{D} p}\left(k_{1 \|}\right) T_{\mathrm{D} p}\left(k_{2 \|}\right) T_{\mathrm{D} p}\left(k_{3 \|}\right) \delta\left(\sum k_{\|}\right) .
\end{aligned}
$$

The three-tachyon coupling can be read off;

$$
g_{T}=\frac{2}{(4 \log 2)^{3 / 2} \sqrt{\mathcal{K}(2 \pi)^{p-25}\left\langle\Psi_{g} \mid \mathcal{Q} \Psi_{g}\right\rangle}} .
$$

Following [8], the $\mathrm{D} p$-brane tension is given by

$$
\mathcal{T}=\frac{1}{2 \pi^{2} g_{T}^{2}}
$$

so that using eqn. (26) we can recover the standard formula for the ratio of D-brane tensions,

$$
\frac{\mathcal{T}_{p+1}}{\mathcal{T}_{p}}=\frac{1}{2 \pi \sqrt{\alpha^{\prime}}}
$$

where we have restored $\alpha^{\prime}$.

\section{Background $B$-field}

Here we investigate the effect of a constant background $B$-field, and compute the tension as in the previous section. The worldsheet CFT is given by the action 21]

$$
S=\frac{1}{2} \int \mathrm{d}^{2} z\left(g_{\mu \nu} \partial_{\alpha} X^{\mu} \partial_{\beta} X^{\nu} h^{\alpha \beta}-B_{i j} \partial_{\alpha} X^{i} \partial_{\beta} X^{j} \epsilon^{\alpha \beta}\right),
$$

where $\mu$ and $\nu$ run from 0 to 25, while for simplicity $i$ and $j$ will take only the values 24 and 25. That is, the $B$-field has non-zero components only in these two directions. This action 
leads us to the usual Neumann boundary conditions for the first 24 directions, while for the last two, we have

$$
\left.\left(\delta_{i j} \partial_{n}+B_{i j} \partial_{t}\right) X^{j}(z)\right|_{\partial \Sigma}=0
$$

In the following, we concentrate on these two directions and often omit explicit indices. The Green function on the unit disc is given by

$$
\langle X(z) X(w)\rangle_{\text {Disc }}=-\log |z-w|-\frac{1}{2}\left(\frac{1+B}{1-B}\right) \log \left(z-\frac{1}{\bar{w}}\right)-\frac{1}{2}\left(\frac{1-B}{1+B}\right) \log \left(\bar{z}-\frac{1}{w}\right) .
$$

This can be written [21] in terms of the open-string metric $G$ and the non-commutativity parameter $\theta$. These are related to the $B$-field by

$$
\theta=-\frac{1}{1+B} B \frac{1}{1-B} \quad \text { and } \quad G=\frac{1}{1+B} 1 \frac{1}{1-B}
$$

On the boundary, $|z|=1$, the correlator (31) becomes

$$
\left\langle X^{i}\left(e^{i \phi}\right) X^{j}\left(e^{i \phi^{\prime}}\right)\right\rangle_{\partial \mathrm{D}}=-G^{i j} \log \left(2 \sin \frac{\phi-\phi^{\prime}}{2}\right)^{2}+\frac{i}{2} \theta^{i j} \epsilon\left(\phi-\phi^{\prime}\right) .
$$

\section{$4.1 \quad(B, g)$-Parametrisation}

In section 3, to define the D-brane state $|\mathrm{D} p\rangle$ in eqn. (5) and the tachyon perturbation $|\chi(k)\rangle$ in eqn. (11), we inserted operators $\sigma^{ \pm}$to change the boundary condition to Dirichlet on part of the cylinder $C_{n}$. We can accommodate the new boundary condition (30) by instead inserting new operators $\beta^{ \pm}$at the same positions. Thus on the cylinder $C_{n}$, we have effectively a Neumann boundary condition on the region $-\pi / 4<z<\pi / 4$, and eqn. (30) will apply to the rest of the boundary. We will again absorb the various singular factors due to coincident $\beta^{+}$and $\beta^{-}$operators into the states.

Analogous to eqn. (5) we define the sliver state in the presence of a $B$-field as

$$
\left\langle\Xi_{B} \mid \phi\right\rangle=\lim _{n \rightarrow \infty} \mathcal{N}\langle f \circ \phi(0)\rangle_{C_{n}}^{\beta},
$$

where now the superscript indicates that we have inserted the operators $\beta^{ \pm}$in the 24 - and 25-directions.

We now define a tachyon perturbation in the presence of the $B$-field as

$$
\left|T_{B}\right\rangle=\int \mathrm{d} k n^{-k_{\sharp}^{2}-k_{b} \frac{1}{1-B^{2}} k_{b}} T_{B}(k)\left|\chi_{B}(k)\right\rangle,
$$


where $k_{\sharp}$ refers to the first 24 directions, and $k_{b}$ contains the last two components of $k . T_{B}(k)$ is the tachyon field, and we have used the notation

$$
k_{b} \frac{1}{1-B^{2}} k_{b} \equiv k_{b}^{\mu}\left(\frac{1}{1-B^{2}}\right)_{\mu \nu} k_{b}^{\nu} .
$$

As in the previous section, we can write down the solution to the linearised equation of motion for the perturbation $\chi_{B}(k)$;

$$
\left\langle\chi_{B}(k) \mid \phi\right\rangle=\lim _{n \rightarrow \infty} \mathcal{N} n^{2 k_{\sharp}+2 k_{b} \frac{1}{1-B^{2}} k_{b}}\left\langle f \circ \phi(0) e^{i k \cdot X}(n \pi / 4)\right\rangle_{C_{n}}^{\beta \perp},
$$

where this time we have inserted the operators $\beta^{ \pm}$to impose the $B$-field boundary condition. Exactly analogously to the case of the $\mathrm{D} p$-brane, this state can be shown to satisfy the following,

$$
\begin{aligned}
\left\langle\chi_{B}(k) \mid \chi_{B}\left(k^{\prime}\right)\right\rangle= & \mathcal{K} \sqrt{\operatorname{det}(1+B)} \lim _{n \rightarrow \infty} n^{2 k_{\sharp}^{2}+2 k_{b} \frac{1}{1-B^{2}} k_{b}} 2^{2 k_{\sharp}^{2}+2 k_{b} \frac{1}{1-B^{2}} k_{b}} \\
& (2 \pi)^{26} 4 \delta\left(k_{\sharp}+k_{\sharp}^{\prime}\right) \operatorname{det} \frac{1}{1-B^{2}} \delta\left(\left(k_{b}+k_{b}^{\prime}\right) \operatorname{det} \frac{1}{1-B^{2}}\right) \\
\left\langle\Xi_{B} * \chi_{B}(k) \mid \chi_{B}\left(k^{\prime}\right)\right\rangle= & \mathcal{K} \sqrt{\operatorname{det}(1+B)} \lim _{n \rightarrow \infty} n^{2 k_{\sharp}^{2}+2 k_{b} \frac{1}{1-B^{2}} k_{b}} 2^{k_{\sharp}^{2}+k_{b} \frac{1}{1-B^{2}} k_{b}} \\
& (2 \pi)^{26} 4 \delta\left(k_{\sharp}+k_{\sharp}^{\prime}\right) \operatorname{det} \frac{1}{1-B^{2}} \delta\left(\left(k_{b}+k_{b}^{\prime}\right) \operatorname{det} \frac{1}{1-B^{2}}\right),
\end{aligned}
$$

so that off-shell

$$
\left\langle\chi_{B}(k) \mid \chi_{B}\left(k^{\prime}\right)\right\rangle=2^{k_{\sharp}^{2}+k_{b} \frac{1}{1-B^{2}} k_{b}-1}\left\langle\chi_{B}(k) * \Xi_{B}+\chi_{B}(k) * \Xi_{B} \mid \chi_{B}\left(k^{\prime}\right)\right\rangle .
$$

We see that the on-shell condition is now

$$
k_{\sharp}^{2}+k_{b} \frac{1}{1-B^{2}} k_{b}=1,
$$

to be compared with eqn. (16). In the limit of a large $B$-field, $1 /\left(1-B^{2}\right) \rightarrow 0$, and we recover the case of a D23-brane with on-shell tachyon condition $k_{\sharp}^{2}=k_{\|(23)}^{2}=1$. In the $B \rightarrow 0$ limit, $1 /\left(1-B^{2}\right) \rightarrow 1$ and a D25-brane obtains, with $k_{\sharp}^{2}+k_{b}^{2}=k_{\|(25)}^{2}=1$. Substituting eqn. (38) into the quadratic part of the action as before, and considering the large- $B$ limit we are led to define a 24-dimensional tachyon field by

$$
\widetilde{T_{B}}\left(k_{\sharp}\right) \equiv \sqrt{\mathcal{K}(2 \pi)^{2}\left\langle\Psi_{g} \mid \mathcal{Q} \Psi_{g}\right\rangle 4 \log 2 \sqrt{\operatorname{det}(1+B)}} \int \mathrm{d}^{25-p} k_{b} T_{B}(k) .
$$


The tension of the resulting $\mathrm{D} p$-brane (now taking $k_{b}$ to represent $25-p$ dimensions rather than two) is thus given by

$$
\frac{\mathcal{T}_{p}^{B}}{\mathcal{T}_{25}}=\sqrt{\operatorname{det}(1+B)}(2 \pi)^{(25-p)} \alpha^{\prime(25-p) / 2} .
$$

\section{$4.2(G, \theta)$-Parametrisation}

Defining the tachyon, in the presence of the open-string metric $G$, we write

$$
\left|T_{N C}\right\rangle=\int \mathrm{d} k n^{k^{2}} T_{N C}(k)\left|\chi_{N C}(k)\right\rangle
$$

where since $G$ is the effective metric, it is understood that $k^{2} \equiv k^{\mu} G_{\mu \nu} k^{\nu}$. Looking at eqn. (32), we see that this normalisation is the same as that used in eqn. (35).

The perturbation is now of the form

$$
\left\langle\chi_{N C}(k) \mid \phi\right\rangle=\lim _{n \rightarrow \infty} \mathcal{N} n^{k^{2}}\left\langle f \circ \phi(0) e^{i k \cdot X}(n \pi / 4)\right\rangle_{C_{n}}^{\beta \perp},
$$

and the correlators are found to be

$$
\begin{aligned}
\left\langle\chi_{N C}(k) \mid \chi_{N C}\left(k^{\prime}\right)\right\rangle & =\mathcal{K} \sqrt{\operatorname{det} G} \lim _{n \rightarrow \infty} n^{k^{2}} 2^{k^{2}}(2 \pi)^{26} 4 \delta\left(k+k^{\prime}\right) \\
\left\langle\Xi_{N C} * \chi_{N C}(k) \mid \chi_{N C}\left(k^{\prime}\right)\right\rangle & =\mathcal{K} \sqrt{\operatorname{det} G} \lim _{n \rightarrow \infty} n^{2 k^{2}} 2^{k^{2}}(2 \pi)^{26} 4 \delta\left(k+k^{\prime}\right),
\end{aligned}
$$

with off-shell equation of motion

$$
\left\langle\chi_{N C}(k) \mid \chi_{N C}\left(k^{\prime}\right)\right\rangle=2^{k^{2}-1}\left\langle\chi_{N C}(k) * \Xi_{N C}+\chi_{N C}(k) * \Xi_{N C} \mid \chi_{N C}\left(k^{\prime}\right)\right\rangle .
$$

We now have a 'non-commutative' on-shell condition

$$
k^{\mu} G_{\mu \nu} k^{\nu}=1
$$

where we have written $G$ explicitly. We substitute eqn. (46) into the quadratic part of the action and re-define the tachyon field $T_{N C}$;

$$
\widetilde{T_{N C}} \equiv \sqrt{\mathcal{K}\left\langle\Psi_{g} \mid \mathcal{Q} \Psi_{g}\right\rangle \sqrt{\operatorname{det} G} 4 \log 2} T_{N C}
$$


With this, the tachyon action becomes

$$
\begin{aligned}
S= & S^{(2)}+S^{(3)} \\
= & -\frac{1}{2}(2 \pi)^{26} \int \mathrm{d} k\left(k^{2}-1\right) \widetilde{T_{N C}}(k) \widetilde{T_{N C}}(-k) \\
& -\frac{1}{3} \frac{2(2 \pi)^{26}}{(4 \log 2)^{3 / 2} \sqrt{\mathcal{K}\left\langle\Psi_{g} \mid \mathcal{Q} \Psi_{g}\right\rangle \sqrt{\operatorname{det} G}}} \\
& \quad \int \mathrm{d} k_{1,2,3} \widetilde{T_{N C}}\left(k_{1}\right) * \widetilde{T_{N C}}\left(k_{2}\right) * \widetilde{T_{N C}}\left(k_{3}\right) \delta\left(\sum k\right)
\end{aligned}
$$

where $*$ represents the Moyal product. We identify the cubic tachyon coupling to find the tension of this non-commutative D25-brane

$$
\mathcal{T}_{25}^{N C}=\sqrt{\operatorname{det} G} \mathcal{T}_{25}
$$

Finally, we identify the ratio

$$
\frac{\mathcal{T}_{23}^{B}}{\mathcal{T}_{25}^{N C}}=(2 \pi)^{2} \alpha^{\prime} \sqrt{\frac{\operatorname{det}(1+B)}{\operatorname{det} G}}=\frac{(2 \pi)^{2} \alpha^{\prime}}{(\operatorname{det} G)^{1 / 4}},
$$

as demonstrated in the papers [16] and [17].

\section{Discussion}

In 13 it was proposed that in Vacuum String Field Theory a D-brane of arbitrary dimension may be represented by inserting boundary condition-changing twist operators into the CFT description of the sliver state in the directions transverse to the D-brane. We have calculated the ratio of tensions of D-branes using this approach and obtain eqn. (28), in agreement with

standard string theory. Similar calculations in VSFT have recently been done in [16] and [17], using the algebraic oscillator approach.

Additionally, in section 1 turning on a constant $B$-field in some directions can be represented in similar fashion, by the insertion of operators $\beta^{ \pm}$which suitably modify the boundary condition. In this way, we have shown that a non-commutative D-brane solution can also be constructed from the sliver state, for generic constant $B$-field.

Of course, for the case of vanishing $B$-field, we recover the D25-brane solution, while in the large- $B$ limit, the boundary conditions become Dirichlet, and we find agreement with 
our tension calculations for the lower-dimensional D-brane in section 3. Although for finite $B$ there is no clear way to re-define the tachyon field, in the D25-brane case we calculated the tension of a non-commutative D25-brane.

There was some discussion in [20], [15] and [18 as to whether the sliver state represents a single or multiple D-brane state. Although the quantities calculated here do not shed light on this question since they are ratios of tensions, they do provide further evidence that we are in fact dealing with a D-brane state, and that the proposals of [13] describe the correct way to represent D-branes in VSFT.

\section{Acknowledgements}

P.M. and Y.Y. are grateful to Simon Fraser University for Graduate Fellowship support. R.R. would like to thank Simon Fraser University for kind hospitality. This work was supported in part by an operating grant from the Natural Sciences and Engineering Research Council of Canada.

\section{Appendix}

Here we show the method of calculation of the correlators used in the main text; specifically, we make an example of eqn. (13). The other correlators used in the main text may be calculated in a similar way, and the details of the limiting procedure may be found in [18].

Starting with eqn. (12), we map $C_{m+n-2}$ to the unit disc using $z \rightarrow e^{\frac{4 i z}{m+n-2}}$. We note that since the zero mode of the expansion for $X(z)$ contributes to the conformal dimension of the tachyon vertex operator, and this is absent in the Dirichlet case, the conformal dimension of $e^{i k \cdot X}$ (which is of course normal-ordered) is $k_{\|}^{2} / 2$. On the disc, then,

$$
\left\langle\chi(k) \mid \chi\left(k^{\prime}\right)\right\rangle=\mathcal{N}^{2} \lim _{n, m \rightarrow \infty} n^{2 k_{\|}^{2}} m^{k^{\prime 2} \|}\left(\frac{4 i}{m+n-2}\right)^{k_{\|}^{2}}\left(\frac{-4 i}{m+n-2}\right)^{k^{\prime 2}}\left\langle e^{i k \cdot X}(1) e^{i k^{\prime} \cdot X}(-1)\right\rangle_{\operatorname{Disc}}^{\sigma \perp} .
$$

The zero mode we mentioned above also produces a $\delta$-function in the correlator, 22 giving

$$
\left\langle e^{i k \cdot X}(z) e^{i k^{\prime} \cdot X}(w)\right\rangle_{\mathrm{D}}^{\sigma \perp}=e^{i k \cdot k^{\prime}\langle X(z) X(w)\rangle} \delta\left(k_{\|}+k_{\|}^{\prime}\right) e^{i x_{0} \cdot\left(k_{\perp}+k_{\perp}^{\prime}\right)}
$$


for the tachyon propagator. The $X$-propagator is given by

$$
\langle X(z) X(w)\rangle_{D}=-\log |z-w| \pm \log \left|z-\frac{1}{\bar{w}}\right|
$$

where for Dirichlet and Neumann directions the + and - sign is used, respectively. The tachyon correlator on the boundary is thus

$$
\left\langle e^{i k \cdot X}\left(e^{i \theta}\right) e^{i k^{\prime} \cdot X}\left(e^{i \theta^{\prime}}\right)\right\rangle_{\operatorname{Disc}}^{\sigma \perp}=\frac{1}{(2 \epsilon)^{2 h}}\left(2 \sin \frac{\theta-\theta^{\prime}}{2}\right)^{2 k_{\|} \cdot k_{\|}^{\prime}} \delta\left(k_{\|}+k_{\|}^{\prime}\right) e^{i x_{0} \cdot\left(k_{\perp}+k_{\perp}^{\prime}\right)}
$$

where $x_{0}$ is the Dirichlet boundary condition. Substituting this into eqn. (A-1) we have (We absorb factors of $\frac{1}{(2 \epsilon)^{2 h}}$ into the state $\chi(k)$.)

$$
\begin{aligned}
\left\langle\chi(k) \mid \chi\left(k^{\prime}\right)\right\rangle= & \mathcal{N}^{2} \lim _{n, m \rightarrow \infty} n^{2 k_{\|}^{2}} m^{2 k^{\prime}} 2^{-2 k_{\|}^{2}}\left(\frac{4 i}{m+n-2}\right)^{k_{\|}^{2}}\left(\frac{-4 i}{m+n-2}\right)^{k_{\|}^{\prime 2}} \\
& \delta\left(k_{\|}+k_{\|}^{\prime}\right) e^{i x_{0} \cdot\left(k_{\perp}+k_{\perp}^{\prime}\right)} .
\end{aligned}
$$

Taking the $m \rightarrow \infty$ limit, we end up with the result

$$
\left\langle\chi(k) \mid \chi\left(k^{\prime}\right)\right\rangle=\mathcal{K} \lim _{n \rightarrow \infty} n^{2 k_{\|}^{2}} 2^{2 k_{\|}^{2}}(2 \pi)^{26} \delta\left(k_{\|}+k_{\|}^{\prime}\right) e^{i x_{0} \cdot\left(k_{\perp}+k_{\perp}^{\prime}\right)}
$$

\section{References}

[1] Edward Witten, Non-Commutative Geometry and String Field Theory, Nucl. Phys. B268 (1986) 253-294.

[2] David J. Gross and Antal Jevicki, Operator Formulation of Interacting String Field Theory (I), Nucl. Phys B283 (1987) 1.

[3] David J. Gross and Antal Jevicki, Operator Formulation of Interacting String Field Theory (II), Nucl. Phys B287 (1987) 225.

[4] V. Alan Kostelecký and Robertus Potting, Analytical construction of a nonperturbative vacuum for the open bosonic string, Phys. Rev. D63 (2001) 046007.

[5] David J. Gross and Washington Taylor, Split string Field Theory I, JHEP 0108 (2001) 009. 
[6] David J. Gross and Washington Taylor, Split string Field Theory II, JHEP 0108 (2001) 010.

[7] Ashoke Sen, Descent Relations among Bosonic D-branes, Int. J. Mod. Phys. A14 (1999) 4061.

[8] Ashoke Sen, Universality of the Tachyon Potential, JHEP 9912 (1999) 027.

[9] Leonardo Rastelli and Barton Zwiebach, Tachyon Potentials, Star Products and Universality, JHEP 0109 (2001) 038.

[10] Leonardo Rastelli, Ashoke Sen and Barton Zwiebach, String Field Theory Around the Tachyon Vacuum, hep-th/0012251.

[11] Leonardo Rastelli, Ashoke Sen and Barton Zwiebach, Classical Solutions in String Field Theory Around the Tachyon Vacuum, hep-th/0102112.

[12] Leonardo Rastelli, Ashoke Sen and Barton Zwiebach, Half-Strings, Projectors, and Multiple D-branes in Vacuum String Field Theory, JHEP 0111 (2001) 035.

[13] Leonardo Rastelli, Ashoke Sen and Barton Zwiebach, Boundary CFT Construction of D-branes in Vacuum String Field Theory, JHEP 0111 (2001) 045.

[14] Leonardo Rastelli, Ashoke Sen and Barton Zwiebach, Vacuum String Field Theory, hep-th/0106010.

[15] Leonardo Rastelli, Ashoke Sen and Barton Zwiebach, A Note on a Proposal for the Tachyon State in Vacuum String Field Theory, hep-th/0111153.

[16] L. Bonora, D. Mamone and M. Salizzoni, B field and squeezed states in Vacuum String Field Theory, hep-th/0201060.

[17] Kazumi Okuyama, Ratio of Tensions from Vacuum String Field Theory, hep-th/0201136.

[18] Radoslav Rashkov and K. Sankaran Viswanathan, A Note on the Tachyon State in Vacuum String Field Theory, hep-th/0112202.

[19] Partha Mukhopadhyay, Oscillator Representation of the BCFT Construction of Dbranes in Vacuum String Field Theory, hep-th/0110136. 
[20] Hiroyuki Hata and Teruhiko Kawano, Open String States around a Classical Solution in Vacuum String Field Theory, hep-th/0108150.

[21] Nathan Seiberg and Edward Witten, String Theory and Noncommutative Geometry, JHEP 9909 (1999) 032.

[22] Joseph Polchinski, String Theory, Cambridge University Press 1998. 